\title{
funciones ejecutivas en niños con trastorno del lenguaje: algunos antecedentes desde la neuropsicología*
}

\author{
Executive functions in Children with Language Disorder: \\ Some Background from neuropsychology \\ Funções executivas em crianças com transtorno da linguagem: \\ alguns antecedentes desde a neuropsicologia
}

\author{
Nancy Lepe-Martínez ${ }^{* *}$ \\ Universidad Católica del Maule, Talca, Chile. \\ Claudia P. Pérez-Salas*** \\ Universidad de Concepción, Concepción, Chile. \\ Cristian A. Rojas-Barahona**** \\ Pontificia Universidad Católica de Chile, Santiago, Chile. \\ Carlos Ramos-Galarza***** \\ Pontificia Universidad Católica del Ecuador, Quito, Ecuador.
}

Doi: http://dx.doi.org/10.12804/revistas.urosario.edu.co/apl/a.5609

\section{Resumen}

El trastorno del lenguaje es un problema de la comunicación con alta incidencia y prevalencia en la población escolar, el cual afecta negativamente el desempeño académico, y el desarrollo social y afectivo de los niños que lo padecen. Existe evidencia de que en este trastorno se encuentran afectadas funciones ejecutivas (FE), además de los componentes del lenguaje (fonológico, morfosintáctico, semántico y pragmático), los cuales se han estudiado tradicionalmente. Este artículo tiene por objetivo caracterizar las principales FE que se encuentran afectadas en el trastorno del lenguaje. Para ello, se trabajó con un método de revisión narrativa. Se

* Esta investigación contó con el apoyo del Fondo Nacional de Ciencia y Tecnología (Fondecyt-Conicyt) del proyecto $\mathrm{N}^{\circ} 1151262$.

** Nancy Lepe-Martínez, Facultad de Ciencias de la Educación, Universidad Católica del Maule, Talca, Chile. Doctorado en Psicología, Universidad de Concepción. Becaria Conicyt Chile, Beca de Doctorado Nacional, año académico 2016, N ${ }^{\circ}$ 21160399.

*** Claudia P. Pérez-Salas, Departamento de Psicología, Universidad de Concepción, Concepción, Chile.

**** Cristian A. Rojas-Barahona, Facultad de Educación, Pontificia Universidad Católica de Chile, Santiago, Chile.

***** Carlos Ramos-Galarza, Facultad de Psicología, Pontificia Universidad Católica del Ecuador, Quito, Ecuador.

La correspondencia relativa a este artículo debe ser enviada a Nancy Lepe-Martínez, Avenida San Miguel No 3605, Facultad de Ciencias de la Educación, Universidad Católica del Maule, Talca, Chile. Correo electrónico: nlepe@ucm.cl

Cómo citar este artículo: Lepe-Martínez, N., Pérez-Salas C.P., Rojas-Barahona, C. A. \& Ramos-Galarza, C. (2018). Funciones ejecutivas en niños con trastorno del lenguaje: algunos antecedentes desde la neuropsicología. Avances en Psicología Latinoamericana, 36(2), 389-403. DOI: http://dx.doi.org/10.12804/revistas.urosario.edu.co/ap1/a.5609 
realizó una revisión del desarrollo del lenguaje desde la primera infancia, del trastorno del lenguaje y de FE. Los estudios reportan que la principal FE afectada en el trastorno del lenguaje es la memoria de trabajo, y se presentan algunos déficits en atención, flexibilidad cognitiva y control inhibitorio. Finalmente, se discute el rol de las FE en el trastorno del lenguaje y las implicancias que esto tiene tanto para la evaluación como para la generación de apoyos pedagógicos pertinentes.

Palabras clave: trastorno del lenguaje, funciones ejecutivas, memoria de trabajo.

\section{fibstract}

Language disorder is a communication disorder with high incidence and prevalence in the school population and wich negatively affects the academic performance, and the social and emotional development of the children suffering this condition. There is evidence that in this disorder the executive functions (EF) are affected, in addition to the language (phonological, morphosyntactic, semantic and pragmatic) components, which have been traditionally studied. This article aims to characterize the main $\mathrm{EF}$ that are affected in language disorder. For this purpose, we worked with a method of narrative review. A review of language development from early childhood, language disorder and EF was made. Studies report that the main EF affected in the language disorder are working memory, some deficits in attention, cognitive flexibility and inhibitory control. Finally, the role of the EF in language disorder is discussed and the implications that this has both for the evaluation and for the generation of pertinent pedagogical supports.

Keywords: language impairment, Executive functions, Working memory.

\section{Resumo}

O transtorno da linguagem, é um transtorno da comunicação com alta incidência e prevalência na população escolar e afeta negativamente o desempenho acadêmico, desenvolvimento social e afetivo das crianças que o padecem. Existe evidência que neste transtor- no encontram-se afetadas funções executivas (FE), para além dos componentes da linguagem (fonológico, morfossintático, semântico e pragmático), os que se têm estudado tradicionalmente. Nesta linha, este artigo tem por objetivo caracterizar as principais FE que se encontram afetadas no transtorno da linguagem. Para isso, se trabalhou com um método de revisão narrativa. Se realizou uma revisão do desenvolvimento da linguagem desde a primeira infância, do transtorno da linguagem é a memória de trabalho, também se apresentariam alguns déficits em atenção, flexibilidade cognitiva e controle inibitório. Finalmente, discute-se o papel das FE no transtorno da linguagem e as implicâncias que isto tem tanto para a avaliação quanto para a geração de apoios pedagógicos pertinentes.

Palavras-chave: trastorno da linguagem, funções executivas, memória de trabalho.

\section{Introducción}

La Asociación Americana de Psiquiatría (APA, 2013) define el trastorno del lenguaje como un problema de la comunicación que se manifiesta en dificultades persistentes en la adquisición y uso del lenguaje que no pueden ser explicadas por factores personales o sociales de relevancia. Los niños que presentan trastorno del lenguaje tienen un adecuado desarrollo sensorial, emocional y social, en presencia de oportunidades lingüísticas potenciadoras y sin déficit intelectual. Este trastorno tiene una alta prevalencia en la población infantil entre 3 a 6 años (Acosta, Moreno \& Axpe, 2012; Conti-Ramsden, Clair, Pickles \& Durkin, 2012; Peterson \& Gardner, 2011; Von Keyserlink, Castro \& Carrasco, 2013).

Los estudios sobre este trastorno se han agrupado principalmente en torno a dos posturas o enfoques: (1) un enfoque lingüístico (Peterson \& Gardner, 2011), que lo considera un problema específico, en el que las dificultades centrales radican en los componentes del lenguaje, es decir, fonológico, morfosintáctico, semántico y prag- 
mático; y (2) el enfoque de procesamiento de la información (Bermeosolo, 2012; Hincapié et al., 2007; Martínez, Herrera, Valle \& Vásquez, 2011; Mongomery \& Windsor, 2007), que lo considera un problema general de la cognición, encontrándose afectados los componentes tanto lingüísticos como no lingüísticos (Mendoza, 2012).

Los estudios sobre el tema, desde una perspectiva cognitiva más general, han demostrado que existen FE deficientes en niños que presentan este trastorno. Las FE pueden ser entendidas como procesos cognitivos de alta complejidad que permiten la organización temporal tanto del comportamiento, como del lenguaje y el razonamiento (Fuster, 2008) para la resolución de problemas internos y externos (Delgado-Mejías \& Etchepareborda, 2013). Las FE están compuestas por habilidades de control atencional, memoria de trabajo, planeación, integración temporal, toma de decisiones y control inhibitorio (Fuster, 2008), entre otras.

En dicho marco de desarrollo del trastorno del lenguaje, este artículo tiene por objetivo caracterizar las FE que se encuentran afectadas en el trastorno del lenguaje, con la finalidad de aportar una explicación a sus causas desde lo neuropsicológico y no solo desde lo lingüístico, para así contribuir con información que permita mejorar la detección y la intervención pedagógica del trastorno.

\section{Método}

Para el logro del objetivo propuesto, se usó el método de revisión narrativa (Letelier, Manríquez $\&$ Rada, 2005), cuyo propósito es entregar un estado general de los hallazgos sobre las FE que se ven afectadas en el trastorno del lenguaje. Para ello, se registran las distintas fuentes en donde se realiza la búsqueda, que en este caso fue mediante la revisión de las bases de datos Scopus, Web of Sciencie y Google académico, en los últimos 12 años (2006-2018). La selección del periodo mencionado se debe a que hasta el 2005 se observa un promedio anual de 7 artículos publicados en el tema (véase la base Scopus), y desde el 2006 se nota un crecimiento exponencial, pasando a un promedio de 42 artículos publicados al año.

Los criterios de inclusión de investigaciones teóricas o aplicadas fue que analizaran las FE afectadas en el trastorno del lenguaje. Las palabras clave utilizadas fueron "funciones ejecutivas en niños" y "trastornos específicos del lenguaje", fue usado este término porque así se ha clasificado tradicionalmente a este trastorno.

\section{Desarrollo del lenguaje}

El aprendizaje del lenguaje se define como el manejo progresivo de símbolos que se adquirieren según un curso que está mediado por factores tanto innatos como ambientales. Los primeros le permiten a un niño adquirir el lenguaje independientemente de la cultura en la que esté inserto. Por su parte, los factores ambientales interactúan de manera dinámica con los factores innatos, lo que permite que el curso de desarrollo del lenguaje pueda experimentar ciertas variaciones (LópezOrnat, 2011; Martínez \& Romero, 2007; Rodríguez, 2012), lo cual explica las diferencias individuales que se pueden observar incluso en niños que presentan un adecuado desarrollo lingüístico.

El curso de desarrollo del lenguaje oral se inicia desde el nacimiento. En esta etapa el niño presta más atención al lenguaje que ha escuchado desde antes de nacer que a uno distinto a este. Desde pequeños los niños atienden al que habla, comienzan a ser sensibles a los tonos de habla de su lengua, toman turnos en el balbuceo y a los 11 meses ya reconocen aproximadamente 50 palabras.

En los inicios del desarrollo del lenguaje, los gestos permiten la comunicación y la interacción con el medio ambiente antes que sea posible el lenguaje oral. Una investigación en el área demuestra que los gestos preceden a la palabra en la intención comunicativa y luego la acompañan. Gestos y palabras se apoyan mutuamente con mayor intensidad en la primera infancia, siendo menor en la vida adulta, 
aunque dicha interacción colaborativa se mantiene a lo largo de la vida (Rodríguez, 2012).

El uso de gestos simbólicos funciona como un andamiaje que facilita en los bebés el desarrollo del lenguaje oral. Estos pueden aumentar su vocabulario con apoyo de gestos que acompañan el lenguaje oral en comparación a otros bebés con los que no se usan gestos. El uso de gestos permite mejorar la atención conjunta sobre el lenguaje, además de acercar el referente al sonido de las palabras ya que los gestos simbólicos permiten representar de manera física la palabra, presentando así simultáneamente su forma y significado, lo que facilita la compresión y la retención del concepto (Goodwyn, Acredolo \& Brown, 2000).

El desarrollo fonológico del lenguaje se inicia con el balbuceo, que comienza a los seis meses aproximadamente, y desde el primer año de vida comienza la producción de palabras de uso común, que son pronunciadas de manera simple y significan cosas bien concretas para un niño. Por su parte, el desarrollo semántico que se inicia con el manejo de vocabulario simple y que hace referencia a objetos concretos más que abstractos comienza su desarrollo en los primeros años de vida y se incrementa significativamente en los próximos años preescolares. En tanto, el manejo de la morfología y la sintaxis se inicia hacia los dos años cuando el niño maneja al menos 50 palabras. En esta etapa combina dos palabras para producir un lenguaje telegráfico pero con un significado completo para el niño (por ejemplo, "mamá leche"). Progresivamente incorpora artículos y tiempos verbales, hasta llegar a la etapa escolar con un lenguaje similar al de un adulto (Gleason \& Ratner, 2010).

Como es posible establecer un curso de desarrollo "típico" del lenguaje, independiente de la cultura de procedencia (Gleason \& Ratner, 2010), que se despliega generalmente sin problemas en los niños que están insertos en entornos lingüísticos, es posible entonces reconocer a los niños que presentan un desarrollo que no sigue esa trayectoria, lo que se puede considerar un desarrollo atípico.
Dentro de este grupo de niños que no sigue ese patrón típico de desarrollo del lenguaje se encuentran los que muestran trastorno del lenguaje. Ellos no presentan problemas neurológicos, discapacidad intelectual, trastornos de la conducta, ni carencias ambientales de relevancia, sin embargo, no pueden seguir el curso de desarrollo típico del lenguaje por el que transita la mayoría de sus pares (Quintero, Hernández, Verche, Acosta \& Hernández, 2013).

\section{Trastorno del lenguaje}

Este concepto, según DSM-5 (APA, 2013), deja de denominarse trastornos específicos del lenguaje (TEL) y se denomina simplemente trastorno del lenguaje; es un problema de la comunicación que forma parte de los trastornos del desarrollo neurológico. Se define como un conjunto de dificultades persistentes tanto en la adquisición como en la producción del lenguaje, en sus distintas modalidades: hablado, escrito y signos. Sus principales problemas son el vocabulario reducido, las pobres estructuras gramaticales y un deterioro en el discurso. No puede ser explicado por problemas sensoriales, motrices o neurológicos, tampoco por discapacidad intelectual. Las manifestaciones se presentan en la primera infancia y afectan significativamente la comunicación efectiva, los logros académicos o laborales y la participación social (APA, 2013).

La prevalencia del trastorno del lenguaje se estima en un $7 \%$ en Estados Unidos (Peterson \& Gardner, 2011), en un $4 \%$ en Chile (Von Keyserlink, Castro \& Carrasco, 2013) y en un 7,4 \% en la población infantil a nivel mundial (Acosta, 2014). Es un trastorno que presenta una alta persistencia en el tiempo, por lo que es considerado uno de los problemas de la comunicación oral más frecuente en niños de 3 a 7 años, afectando diversas áreas del aprendizaje escolar, como la lectura, la escritura y las matemáticas, con el consiguiente efecto negativo sobre el progreso escolar y el desarrollo socioemocional de quienes lo padecen (Acosta, Moreno \& Axpe, 2012; Conti-Ramsden et al., 2012). 


\section{Desarrollo de las funciones ejecutivas}

Las FE son un constructo amplio que puede ser conceptualizado como un conjunto de procesos cognitivos, afectivos y motivacionales destinados al control consciente del pensamiento (Lezak, 1982; Mauricio, Stelzer, Mazzoni \& Alvarez, 2012; Pineda, 2000; Pistoia, Abad-Mas \& Etchepareborda, 2004), lo que permite la anticipación de la conducta, el establecimiento de metas y la autorregulación tanto de las operaciones mentales como de la conducta para la resolución eficaz de problemas (Pineda, 2000). Las FE se encuentran implicadas en procesos de control afectivo y motivacional, por lo que se las puede clasificar también en FE "frías" y “calientes” (Mauricio et al., 2012): las primeras se relacionan con tareas abstractas y sin un contexto ecológico; las calientes están implicadas en tareas que demandan regulación emocional o requieren de la evaluación del significado motivacional de la tarea.

El desarrollo de las FE es progresivo y secuencial con periodos más intensos de desarrollo y otros más lentos y prolongados. No existe entre los autores plena coincidencia entre las etapas de desarrollo de las FE, pero es posible ver un largo desarrollo posnatal que se inicia alrededor del primer año de vida y culmina en la adultez (Mauricio et al., 2012). La mayoría de las FE comienza su desarrollo tempranamente, lo que permite que a corta edad un niño tenga una conducta regulada ejecutivamente.

Estudios han demostrado que incluso los bebés al año de vida pueden realizar actividades ejecutivas, como mantener representaciones simples durante un lapso breve de tiempo (memoria de trabajo), de manera débil e intermitente. Al año pueden suprimir (control inhibitorio), por algún tiempo al menos, respuestas dominantes (Garcia-Molina, Enseñat-Cantallops, Tirapu-Ustárroz \& Roig-Rovira, 2009); estos serían los primeros rudimentos de una conducta regulada ejecutivamente. El pleno desarrollo se inicia hacia los 4 años de edad cuando un niño es capaz de ejercer cierto control inhibitorio sobre su conducta (Delgado-Mejias \& Etchepareborda, 2013). Entre los 6 y los 8 años, un niño adquiere la capacidad para regular la conducta, fijarse metas (planificación) y anticipar acontecimientos sin la guía de un adulto (Pineda, 2000), todas capacidades requeridas para el éxito escolar y social.

Las primeras FE que se desarrollan en un niño, entre los 0 y los 5 años aproximadamente, son la capacidad de toma de decisiones y el control inhibitorio. Así, un niño de 4 o 5 años de edad es capaz de tomar decisiones respecto de una actividad, y a los 8 años esta competencia es similar a la de un adolescente o la de un adulto. El control inhibitorio que implica el manejo de actividades automáticas se inicia hacia los 4 años de edad (FloresLozano, Castillo-Preciado \& Jiménez-Miramontes, 2014).

Las FE que se desarrollan en los primeros años escolares, entre los 7 y 9 años aproximadamente, son la memoria de trabajo, la flexibilidad mental, la planeación viso-espacial y secuencial, y la memoria estratégica (Flores-Lozano et al., 2014). La memoria de trabajo viso-espacial secuencial alcanza su máximo desarrollo a los 12 años (Luciana \& Nelson, 2002); así mismo, la flexibilidad mental alcanza su máximo desarrollo alrededor de los 12 años de edad (Anderson, 2001) y la capacidad de planeación viso-espacial tiene su mejor desempeño a los 12 años (Levin, Song, Swing-Cobbs \& Roberson, 2001). La capacidad de memoria estratégica se comienza a ejecutar de manera eficiente a partir de los 7 años (Roebers, 2006).

Las últimas FE en desarrollarse, entre los 11 y los 15 años, aproximadamente, son la flexibilidad cognitiva, la cual permite el desarrollo de la fluidez verbal, que facilita el uso de verbos comunes al inicio y más abstractos hacia los 12 años (Ávila, 2004). La capacidad de abstracción se desarrolla de manera progresiva desde los 6 años hasta la adolescencia (Flores-Lázaro \& OstroskySolís, 2008). 
En la actualidad existen antecedentes de que las principales FE a considerar son control inhibitorio, memoria de trabajo y flexibilidad cognitiva (Miyake et al., 2000; Lehto, Juujarvi, Kooistra \& Pulkkinen, 2003; Rojas-Barahona, 2017; Santa-Cruz \& Rosas, 2017).

\section{Funciones ejecutivas deficientes en el trastorno del lenguaje}

Al revisar las FE deficientes en el trastorno del lenguaje, es posible identificar posturas discrepantes. Algunos estudios muestran que los niños con trastorno del lenguaje no tienen afectadas las FE ya que son capaces de planificar sus acciones y actuar flexiblemente (véase, por ejemplo, ArboledaRamírez et al., 2007; Peterson \& Gardner, 2011). Hay otros estudios que dan cuenta de que los niños con trastorno del lenguaje poseen un rendimiento menor en tareas de FE que sus pares sin el trastorno, ya que tendrían un pensamiento menos flexible, y menor capacidad de mantener o cambiar el foco de atención e inhibir estímulos ajenos a la tarea. Todo esto afectaría la compresión y la expresión del lenguaje (véase, por ejemplo, Bermeosolo, 2012; Brigitte, Cuperus, Hendriks \& Verhoeven, 2013; Mendoza, 2012; Quintero et al., 2013).

Por su parte, en los estudios sobre el trastorno del lenguaje también es posible encontrar cierta controversia, porque hay posturas que lo consideran un problema específico del módulo lingüístico y que es independiente de otros factores cognitivos (Peterson \& Gardner, 2011). Según esta perspectiva, se presentarían problemas en la concordancia y en las reglas gramaticales, por un conocimiento limitado de las que rigen el lenguaje, por dificultades en el dominio de estas y por falta de reconocimiento de la obligatoriedad de su uso. Todo lo anterior impactaría negativamente el uso adecuado del lenguaje según códigos prestablecidos (Conde-Guzón, Conde-Guzón, Bartolomé-Albistegui \& Quirós-Expósito, 2009).
Sin embargo, es posible encontrar también creciente evidencia empírica proveniente de estudios específicos (Bermeosolo, 2012; Hincapié et al., 2007; Martínez, Herrera, Valle \& Vásquez, 2011; Montgomery \& Windsor, 2007) y de meta-análisis (Brigitte et al., 2013; Mendoza, 2012) que muestran que las limitaciones que presentan los niños con trastorno del lenguaje no están restringidas a una o unas pocas habilidades lingüísticas, sino que afectan un conjunto de habilidades lingüísticas y no lingüísticas que se relacionan entre sí (Arboleda-Ramírez et al., 2007; Conde-Guzón et al., 2009), por lo que podría ser considerado un trastorno de alcance más general y no centrado solo en variables lingüísticas.

Según esta postura más amplia, que integra las deficiencias lingüísticas con los problemas en $\mathrm{FE}$, es posible indicar que en el trastorno del lenguaje se encuentra afectado el desarrollo fonológico, además de existir problemas sintácticos y semánticos, y dificultades para procesar información lingüística recibida, no por problemas lingüísticos como tal, sino por dificultades en el almacenamiento temporal de la información en la memoria de trabajo, lo que produce un enlentecimiento en el procesamiento de la información en tareas lingüísticas y no lingüísticas. Estas deficiencias en el procesamiento de la información llegarían a afectar también la expresión oral del lenguaje, es decir, la programación del habla, ya que para su correcto funcionamiento es necesario el adecuado desarrollo de secuencias pre-planificadas del habla que se producen mientras se está hablando (Conde-Guzón et al., 2009), las cuales son posibles gracias a la capacidad para retener, organizar y procesar la información, cuya responsable es la memoria de trabajo.

Los problemas no lingüísticos más importantes que acompañan al trastorno del lenguaje desde la perspectiva más general son alteraciones en la memoria de trabajo, la atención, el procesamiento de la información y en habilidades motoras, las cuales son menos eficientes a pesar de contar con 
un coeficiente intelectual no verbal normal, lo que se debería a un déficit cognitivo de procesamiento no lingüístico general (Arboleda-Ramírez et al., 2007). En esta misma línea, existe abundante evidencia de que la memoria de trabajo explicaría de buena manera los orígenes del trastorno, ya que juega un rol importante en la programación, organización, retención y producción del lenguaje (Archibald \& Gathercole, 2007). Los niños con trastorno del lenguaje tienen dificultades no solo en la producción o recepción del lenguaje oral, sino también para atender de modo eficiente a estímulos lingüísticos, y presentan, además, dificultades para codificar las entradas lingüísticas que reciben porque no son capaces de retener ni organizar rápidamente el almacén léxico del que disponen, afectando la adecuada interpretación de la información y, por lo tanto, la producción de lenguaje. Los niños no pueden usar de modo eficiente el repertorio fonológico por dificultades para acceder a este o por deficiencias para decidir en relación con la información almacenada cuál es la más adecuada en la situación comunicativa en la que se encuentran (Buiza-Navarrete, Adrián-Torres \& González-Sánchez, 2007).

Ensayos clínicos realizados por Coelho, Petrucci y Rodrigues (2013) han permitido demostrar que la memoria de trabajo verbal y el procesamiento de información auditiva son deficientes en niños con trastorno del lenguaje. En esta misma línea, otros estudios (Weismer, Evans \& Hesketh, 1999; Marton \& Schwartz, 2003) han demostrado que los niños con trastorno del lenguaje presentan un rendimiento más deficiente que sus pares en memoria de trabajo verbal, sobre todo cuando las tareas requieren simultáneamente el procesamiento y el almacenamiento de la información.

Marton (2008) realizó un estudio que tenía por objetivo medir el procesamiento visoespacial y las FE en niños con trastorno del lenguaje. Concluyó que los niños con trastorno tienen peor desempeño en el control de la atención y la inhibición que aquellos con desarrollo típico del lenguaje, lo que impactaría el procesamiento verbal y visoespacial, y la memoria de trabajo de estos niños. Otro estudio realizado por Marton, Schwartz, Farkas y Katsnelson (2006) comparó la memoria de trabajo de niños húngaros con niños ingleses con y sin trastorno del lenguaje. Observaron que los niños con trastorno de ambas lenguas presentaban peores desempeños en memoria de trabajo que sus pares de la misma edad sin trastorno. El factor central de este peor desempeño radicó en la complejidad sintáctica y morfológica de las estructuras oracionales, ya que en ambos grupos la complejidad de las oraciones afectó significativamente la memoria de trabajo verbal y no así la longitud de los enunciados verbales que se presentaban.

Reichenbach, Bastian, Rohrbach, Gross y Sarrar (2016) midieron algunas FE, como la flexibilidad cognitiva, la inhibición, la velocidad de procesamiento y la memoria a corto plazo, en niños prescolares con trastorno del lenguaje, y observaron que la memoria fonológica a corto plazo es un indicador fiable en el diagnóstico del trastorno del lenguaje, no encontrándose otras FE deterioradas de manera importante en este tipo de niños. En el mismo sentido del estudio anterior, Archibald y Harder (2016), quienes tenían como propósito hacer una medición directa del impacto de la carga de procesamiento de la información sobre la memoria de trabajo, encontraron que los niños con trastorno del lenguaje presentaban una capacidad de almacenamiento fonológico a corto plazo significativamente menor que sus pares sin trastorno, pero no hallaron evidencias de déficit significativo en relación con el recuerdo inmediato. Estos hallazgos implicarían que los niños con trastorno del lenguaje presentarían déficit fonológico a corto plazo pero no habría evidencia de déficit en el componente ejecutivo de la memoria de trabajo.

Estudios recientes indican que los niños con trastorno del lenguaje presentan deficiencias en la memoria de trabajo; esta mayor dificultad la tendrían los niños con trastorno mixto del lenguaje, con consecuencias negativas para su expresión y la 
compresión de material lingüístico de mayor complejidad (Acosta, Ramírez \& Hernández, 2017).
A modo de resumen, se presenta en la tabla 1 una síntesis de algunos de los hallazgos encontrados en los artículos revisados sobre FE deficientes en niños con trastorno del lenguaje.

Tabla 1

Síntesis de estudios que reportan FE deficientes en el trastorno del lenguaje

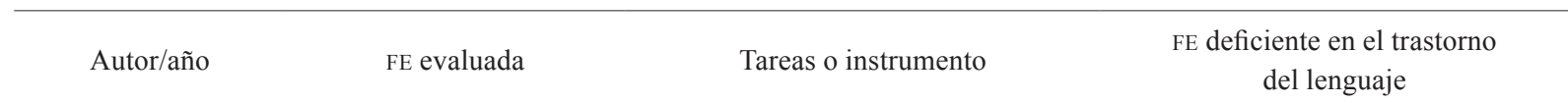

\begin{tabular}{|c|c|c|c|}
\hline $\begin{array}{l}\text { Acosta, Ramírez \& } \\
\text { Hernández (2017) }\end{array}$ & $\begin{array}{l}\text { Memoria de trabajo } \\
\text { verbal. Memoria de } \\
\text { trabajo espacial. } \\
\text { Fluidez verbal. } \\
\text { Fluidez de diseño. } \\
\text { Planificación. Inhibición } \\
\text { y flexibilidad cognitiva. }\end{array}$ & $\begin{array}{l}\text { Subtest de dígitos. Test de span es- } \\
\text { pacial (WMS III). } \\
\text { Test cOWA. } \\
\text { Batería Nepsy. } \\
\text { Test de figuras complejas de Rey. } \\
\text { Test de los cinco dígitos. }\end{array}$ & $\begin{array}{l}\text { Mayor deterioro en la memoria de } \\
\text { trabajo, sobre todo en los niños con } \\
\text { trastorno mixto del lenguaje. }\end{array}$ \\
\hline
\end{tabular}

Archibald \& Harder (2016)

Memoria de trabajo.

Tres tareas complejas de recuperación de letras basadas en el trabajo de Gavens y Barrouillet (2004) que

Memoria de trabajo, el componente ue fonológico es significativamente se diseñaron para imponer cargas de menor que sus pares sin trastorno. procesamiento incremental.

Dimensional Change Card Sort Inhibición

Reichenbach et al. Velocidad de procesamiento.

Memoria a corto plazo.

(DCCS).

Batería BISC

WPPSI-III.

$\mathrm{K}-\mathrm{ABC}$, subprueba retención de dígitos.

Memoria visual y verbal.

Coelho, Petrucci \& Atención.

Rodrigues (2013) Funciones ejecutivas y de percepción visual.
BANC, batería de test para la evaluación neuropsicológica.
Memoria de trabajo fonológica a corto plazo es un indicador fiable en el diagnóstico de niños con trastorno del lenguaje.

Problemas en memoria de trabajo verbal (fonológica) y en el procesamiento de información auditiva.
Memoria de trabajo y

Bermeosolo (2012) memoria de trabajo procedimental a largo plazo.
Deficiencias en memoria de trabajo y memoria de trabajo procedimental a largo plazo en los trastornos del aprendizaje y del lenguaje.

Deficiencias en memoria de trabajo visoespacial; almacenamiento visoespacial y en el control ejecutivo visoespacial.
Brigitte, Cuperus, Hendriks \& Verhoeven (2013)
Memoria de trabajo visoespacial.

Meta-análisis.

Subtest de dígitos modalidad inversa de WISC-IV.

Memoria de trabajo verbal y visoespacial.

Quintero et al. (2013)

Fluidez verbal.
Planificación.

Diseño.

Inhibición y alternancias.
Tarea de localización espacial modalidad inversa de WMS III.

Test de copia y reproducción de figuras complejas de Rey (1987). Test de los cinco dígitos de Sedó (2007).

Controlled Oral Word Association

Test (COWAT) (Benton y Hamster, 1983).
Deficiencias en memoria de trabajo verbal (fonológica) y visoespacial, flexibilidad e inhibición. 


\begin{tabular}{|c|c|c|c|}
\hline Autor/año & FE evaluada & Tareas o instrumento & $\begin{array}{l}\text { FE deficiente en el trastorno } \\
\text { del lenguaje }\end{array}$ \\
\hline $\begin{array}{l}\text { Conde-Guzón et al. } \\
(2009)\end{array}$ & $\begin{array}{l}\text { Revisión de los perfiles } \\
\text { neuropsicológicos de los } \\
\text { niños que manifiestan } \\
\text { diferentes problemas de } \\
\text { lenguaje oral. }\end{array}$ & Revisión sistemática. & $\begin{array}{l}\text { Memoria de trabajo fonológica } \\
\text { y atención; es un denominador } \\
\text { común en los diferentes cuadros de } \\
\text { trastornos del habla infantil. }\end{array}$ \\
\hline Marton (2008) & $\begin{array}{l}\text { Funciones ejecutivas y el } \\
\text { procesamiento visoes- } \\
\text { pacial y la memoria de } \\
\text { trabajo. }\end{array}$ & $\begin{array}{l}\text { En el experimento 1, el rendimiento } \\
\text { de los niños se midió con tres tareas } \\
\text { de procesamiento visoespacial: vi- } \\
\text { sualización del espacio, posición } \\
\text { en el espacio y copia del diseño. } \\
\text { Los estímulos en el experimento } \\
2 \text { fueron dos pruebas neuropsico- } \\
\text { lógicas ampliamente utilizadas: el } \\
\text { Wisconsin Card Sorting Test-64 } \\
\text { (WCST-64) y el examen Tower } \\
\text { of London (TOL). }\end{array}$ & $\begin{array}{l}\text { Memoria operativa (de trabajo). } \\
\text { Las tareas que requieren una mayor } \\
\text { cantidad de control de la atención y } \\
\text { funciones ejecutivas son más difíci- } \\
\text { les para los niños con trastorno que } \\
\text { para sus compañeros sin trastorno. }\end{array}$ \\
\hline $\begin{array}{l}\text { Arboleda-Ramírez } \\
\text { et. al. (2007) }\end{array}$ & $\begin{array}{l}\text { Atención. } \\
\text { Memoria. } \\
\text { Flexibilidad. } \\
\text { Planificación. }\end{array}$ & $\begin{array}{l}\text { Batería Grupo de Neurociencias de } \\
\text { Antioquia. }\end{array}$ & $\begin{array}{l}\text { Dificultades en memoria de trabajo } \\
\text { y atención. }\end{array}$ \\
\hline $\begin{array}{l}\text { Archibald \& } \\
\text { Gathercole (2007) }\end{array}$ & $\begin{array}{l}\text { Memoria de trabajo } \\
\text { fonológica. }\end{array}$ & $\begin{array}{l}\text { Dos tareas experimentales: repe- } \\
\text { tición de series y repetición de } \\
\text { palabras. }\end{array}$ & $\begin{array}{l}\text { Dificultades en memoria de trabajo } \\
\text { fonológica. }\end{array}$ \\
\hline $\begin{array}{l}\text { Buiza-Navarrete, } \\
\text { Adrián-Torres \& } \\
\text { González-Sánchez } \\
\text { (2007) }\end{array}$ & $\begin{array}{l}\text { Atención. } \\
\text { Codificación. } \\
\text { Memoria. } \\
\text { Flexibilidad. }\end{array}$ & $\begin{array}{l}\text { Nueve tareas del sistema de diag- } \\
\text { nóstico neuropsicológico automati- } \\
\text { zado (DIANA). }\end{array}$ & $\begin{array}{l}\text { Dificultades atención y memoria de } \\
\text { trabajo. }\end{array}$ \\
\hline $\begin{array}{l}\text { Marton, Schwartz, } \\
\text { Farkas \& Katsnel- } \\
\text { son }(2006)\end{array}$ & Memoria de trabajo. & $\begin{array}{l}\text { Dos experimentos: en el } 1 \text {, se midió } \\
\text { la precisión del rendimiento con dos } \\
\text { tareas de amplitud lingüística que } \\
\text { incluyeron estímulos con longitud } \\
\text { de frase variable y complejidad } \\
\text { sintáctica. } \\
\text { El experimento } 2 \text { examinó el impac- } \\
\text { to de la longitud de la oración y la } \\
\text { complejidad morfológica en el ren- } \\
\text { dimiento de la memoria de trabajo. }\end{array}$ & $\begin{array}{l}\text { Peores desempeños en memoria de } \\
\text { trabajo. }\end{array}$ \\
\hline
\end{tabular}

Por tanto, según los estudios revisados, como se muestra en la tabla 1 , se puede indicar que la FE que más se ha relacionado con el trastorno del lenguaje es la memoria de trabajo, específicamente el componente fonológico, porque se ha demostrado que esta es la responsable del déficit en el almacenamiento fonológico que presentan los niños con trastorno del lenguaje (Archibald
\& Harder, 2016; Gathercole \& Baddeley, 1990; Reichenbach et al., 2016). Otros estudios han demostrado, además, que el denominador común de los trastorno del lenguaje es la alteración de las FE, específicamente la memoria de trabajo fonológica y verbal a corto plazo (Crespo \& Narbona, 2006; López \& García, 2005). 


\section{Memoria de trabajo y lenguaje}

La memoria de trabajo puede ser entendida como un conjunto de sistemas de almacenamiento que interactúan entre sí para codificar, almacenar y recuperar información (Baddeley, Eysenck \& Anderson, 2010); esto es un aspecto clave en el desarrollo del lenguaje dado que permite mantener activos elementos sintácticos, fonológicos y semánticos para la elaboración y la producción del lenguaje oral.

El modelo más utilizado de memoria de trabajo es el multimodal de Baddeley y Hitch (1974), que está compuesto por cuatro componentes: el bucle fonológico, la agenda visoespacial, el sistema ejecutivo central (Baddeley, Eysenck \& Anderson, 2010) y en el 2000 se incorpora un cuarto componente denominado reten episódico (Baddeley, 2012).

El bucle fonológico está encargado de retener secuencias de elementos acústicos que pueden ser sonidos o lenguaje. Está integrado por un almacén a corto plazo y un proceso de repaso articulatorio; el primero es de capacidad limitada y requiere del repaso vocal o subvocal para recordar la información. El recuerdo de información o palabras es más complejo si las palabras son parecidas fonológicamente y se facilita si son diferentes (Baddeley et al., 2010).

La agenda visoespacial está encargada de mantener elementos y secuencias espaciales y visuales en la memoria de trabajo; permite recordar dónde, lo que hace referencia a aspectos espaciales de la información que se intenta recordar. Este tipo de recuerdo puede ser complementado con gestos que indiquen el lugar físico donde está el objeto y las características del objeto que se intenta recordar (Baddeley et al., 2010).

El ejecutivo central es más un controlador atencional que un sistema de memoria y funciona con dos modos de control: uno semiautomático usado cuando las actividades se transforman en hábitos, por lo que se desarrollan de manera automática, $\mathrm{y}$ otro denominado sistema atencional supervisor, que funciona cuando las respuestas automáticas no son pertinentes al contexto o la tarea, por lo que es necesario generar estrategias para buscar soluciones alternativas. Este último sistema es fundamental para el buen funcionamiento del ejecutivo central porque permite guiar y monitorear la búsqueda de alternativas más adecuadas a la situación o la tarea (Baddeley et al., 2010).

Por último, el retén episódico es un sistema de almacenamiento capaz de mantener bloques de información agrupada en códigos multidimensionales gracias a su capacidad para recibir información proveniente de distintos códigos (sentidos) y recuperarla de manera intencional para realizar un proceso de activación de recuerdos que permita la integración y la construcción de ideas coherentes para su recuerdo y posterior producción (Baddeley et al., 2010).

\section{Discusión}

La búsqueda de una explicación más completa sobre el trastorno del lenguaje se justifica debido a la alta prevalencia de este problema y los efectos negativos que tiene para los niños que lo presentan tanto en su vida académica como en su desarrollo social y emocional. Por esta razón, resulta relevante la realización de estudios que permitan caracterizar las FE que se encuentran afectadas en el trastorno del lenguaje, objetivo del presente trabajo, y así aportar evidencia que complemente lo que ya se sabe en relación con las deficiencias lingüísticas que se presentan en el trastorno. De este modo, se profundiza el conocimiento del trastorno, y así se mejora el tratamiento clínico que se da al trastorno y los apoyos pedagógicos que se brindan a este tipo de niños. Así, se destacan de la revisión realizada algunas ideas centrales que se presentan a continuación.

Las evidencias aportadas por los estudios revisados permiten identificar que una de las principales FE afectadas en el trastorno del lenguaje es la 
memoria de trabajo, principalmente el componente fonológico y, en menor medida, el componente visoespacial. También se presentaría déficits en atención, flexibilidad cognitiva y control inhibitorio, aunque estos se reportan en menor grado en los estudios revisados.

Los déficits en memoria de trabajo afectarían el procesamiento verbal y visoespacial de la información que se recibe, lo cual dificulta significativamente tanto la compresión como la producción del lenguaje, porque, al verse afectada la capacidad de retención y organización fonológica y visoespacial de la información, no es posible la estructuración adecuada de estos elementos, lo que limita la producción y compresión del lenguaje.

Por su parte, el deficiente desempeño del control atencional y la inhibición dificultarían la adecuada recepción e integración de secuencias lingüísticas, lo que afecta la calidad del lenguaje que se produce y el nivel de comprensión que se logra sobre este. Todo lo anterior tendrían graves consecuencias tanto para el desarrollo como para la producción del lenguaje, y sus consabidos efectos negativos sobre el aprendizaje y el desarrollo social y emocional de los niños que lo padecen.

Entender el trastorno del lenguaje como un problema que puede ser explicado tanto por factores lingüístico como por $\mathrm{FE}$ deficientes y contar, además, con claridad sobre cuáles son las FE que se ven más afectadas en el trastorno del lenguaje permite ampliar la mirada sobre los orígenes de este trastorno y, sobretodo, mejorar significativamente su detección y evaluación. Lo anterior redundaría, por un lado, en aspectos diagnósticos a considerar en la evaluación inicial: instrumentos y enfoques de evaluación acordes a la necesidad de evaluación, lo que agregaría valor a esta instancia; y, por otro lado, implicaría apoyos pedagógicos que se hagan cargo de manera integrada en la práctica educativa de los déficits detectados, pudiendo trabajar conjuntamente, por ejemplo, los problemas de memoria fonológica con la capacidad de retención y organización del almacén fonológico para la producción de un fonema particular. Todo lo anterior implicaría un importante avance en el enfoque y forma de acompañar clínica y pedagógicamente a los niños que presentan trastorno del lenguaje.

En un sentido más amplio, entender el trastorno del lenguaje como déficits lingüístico y de FE debería impactar también la política pública, de manera que se pueda disponer de normativas que permita proveer de las herramientas evaluativas y las orientaciones pedagógicas necesarias para la intervención del trastorno del lenguaje, que posibilite un abordaje más integral. Implicaría también revisar la formación de profesores de educación inicial o primaria de manera que puedan contar con la capacitación necesaria para abordar con altos estándares de calidad la estimulación preventiva en los primeros años escolares tanto del lenguaje como de las FE, para brindar a los niños a su cargo oportunidades reales de aprendizaje y desarrollo escolar o de atender eficientemente las necesidades que estos niños presentan una vez que ya son diagnosticados.

Finalmente, tomando en cuenta los antecedentes revisados, se podría conceptualizar de manera provisoria hasta que el conocimiento sobre el tema permita plantear un nuevo concepto acerca del trastorno del lenguaje, como un déficit significativo en el lenguaje expresivo o expresivo-receptivo por dificultades considerables en las FE, especialmente centradas en la memoria de trabajo, que puede afectar tanto la producción como la compresión del lenguaje sin intervención de problemas del desarrollo, ni socioculturales graves.

Dentro de las limitaciones de este trabajo se encuentra la metodología seguida en el artículo, revisión narrativa, que tiene como propósito presentar al lector un estado general del arte pero sin pretensiones de exhaustiva precisión, lo que puede implicar que algunos artículos y autores pudieron haber quedado fuera de esta revisión, los cuales podrían haber aportado información complementaria para el conocimiento del tema. Por tanto, este artículo pretende generar una síntesis 
inicial y actual del tema, en ningún caso se desea que sea una revisión que llegue a una síntesis final o concluyente, sino más bien el inicio de una discusión de los aspectos a tener presente en el trastorno del lenguaje.

\section{Referencias}

Acosta, V. (2014). Efectos de un programa de intervención en el lenguaje en alumnado con trastornos específicos del lenguaje. Revista de Investigación en Educación, 12(1), 92-103. Recuperado de http://webs.uvigo.es/reined/

Acosta, V., Moreno, A. \& Axpe, A. (2012). Efectos de un programa de intervención en el lenguaje sobre el desarrollo del léxico y del procesamiento fonológico en escolares de educación infantil con trastorno específico del lenguaje. Revista de Investigación Educativa, 30(1), 7186. Recuperado de http://revistas.um.es/rie/ article/viewFile/113861/140091

Acosta, V., Ramírez, G. M. \& Hernández, S. (2017). Funciones ejecutivas y lenguaje en subtipos de niños con trastornos específicos del lenguaje. Revista de Neurología, 32(6), 355-362.

American Psychiatric Association (APA) (2013). Statistical manual of mental disorders: DSM-5 (ed.). Washington, DC: American Psychiatric Association.

Anderson, V. (2001). Assessing executive functions in children: biological, psychological and developmental considerations. Pediatric Rehabilitation, 4(3), 119-136. DOI: 10.1080/13638490110091347

Arboleda-Ramírez, A., Lopera-Vásquez, J. P., Hincapié-Henao, L., Giraldo-Prieto, M., Pineda, D. A., Lopera, F. \& Lopera-Echeverri, E. (2007). Trastornos específicos del desarrollo del lenguaje: problema selectivo o generalizado de la cognición. Revista de Neurología, 44(10), 596-600.
Archibald, L. M. \& Gathercole, S. E. (2007). Nonword repetition in specific language impairment: More than a phonological short-term memory deficit. Psychonomic Bulletin \& Review, 14(5), 919-924. DOI: 10.3758/BF03194122

Archibald, L. \& Harder, K. (2016). Rethinking the connection between working memory and language impairment. International Journal of Languaje and Communication Disorder, 51(3), 252-264. DOI: http://doi.org/10.1111/14606984.12202

Ávila, R. (2004). Lo que hacen los niños: verbos y grado escolar. Studia Romanica Posnaniensia, 31,129-146.

Baddeley, A. (2012). Working memory: theories, models, and controversies. Magazine Psychol, 63, 1-29. DOI: 10.1146/annurev-psych-120710-100422

Baddeley, A., Eysenck, M. \& Anderson, M. (2010). Memoria. Madrid: Alianza.

Baddeley, A. \& Hitch, G. (1974). Working memory. Psychology of Learning and Motivation, 8, 47-89. DOI: http://doi.org/10.1016/S00797421(08)60452-1

Bermeosolo, J. (2012). Memoria de trabajo y memoria procedimental en las dificultades especificas del aprendizaje y del lenguaje: algunos hallazgos. Revista Chilena de Fonoaudiología, 11, 57-75. Buiza-Navarrete, J., Adrián-Torres, J. \& González-Sánchez, M. (2007). Marcadores neurocognitivos en el trastorno específico del lenguaje. Revista de Neurología, 44(6), 326-333.

Brigitte, B., Cuperus, J., Hendriks, M. \& Verhoeven, L. (2013). Visuospatial working memory in specific language impairment: a meta-analysis, Research in Developmental Disabilities, 34(9), 2586-2597.

Coelho, S., Petrucci, C. \& Rodrigues, M. (2013). Specific language impairment: a neuropsychological characterization. Paidéia, 23(54), 31-41. DOI: http://dx.doi.org/10.1590/198243272354201305 
Conde-Guzón, P. A., Conde-Guzón M. J., Bartolomé-Albistegui M. T. \& Quirós-Expósito, P. (2009). Perfiles neuropsicológicos asociados a los problemas del lenguaje oral infantil. Revista de neurología, 48(1), 32-38.

Conti-Ramsden, G., Clair, M. C., Pickles, A. \& Durkin, K. (2012). Developmental trajectories of verbal and nonverbal skills in individuals with a history of specific language impairment: from childhood to adolescence. Journal of Speech, Language, and Hearing Research, 55(6), 17161735. DOI: 10.1044/1092-4388(2012/10-0182)

Crespo, N. \& Narbona, J. (2006). Subtipos de trastornos específicos del desarrollo del lenguaje. Perfiles clínicos de una muestra hispanohablante. Revista de Neurología, 43(1), 193-200.

Delgado-Mejias, I. \& Etchepareborda, M. (2013). Trastornos de las funciones ejecutivas. Diagnóstico y tratamiento. Revista de Neurología, 57(1), 95-103.

Flores-Lozano, J., Castillo-Preciado, R. \& Jiménez-Miramontes, N. (2014). Desarrollo de funciones ejecutivas, de la niñez a la juventud. $R e-$ vista Anales de Psicología, 30(2), 463-473. DOI: http://dx.doi.org/10.6018/analesps.30.2.155471

Flores Lázaro, J. C. \& Ostrosky-Solís, F. (2008). Developmental characteristics in category generation reflects different prefrontal cortex maturation. Advances in Psychology Research, 55, 3-13.

Fuster, J. (2008). The prefrontal cortex ( $4^{\mathrm{a}}$ ed.). Londres: Academic Press.

García-Molina, A., Enseñat-Cantallops, A., Tirapu-Ustárroz, J. \& Roig-Rovira, T. (2009). Maduración de la corteza prefrontal y desarrollo de las funciones ejecutivas durante los primeros cinco años de vida. Revista de Neurología, 48(8), 435-440.

Gathercole, S. \& Baddeley, A, (1990). Phonological memory deficits in language disordered children: is there a causal connection? Journal of Memory and Language, 29(3), 336-360.
Goodwyn, S. W., Acredolo, L. P. \& Brown, C. A. (2000). Impact of symbolic gesturing on early language development. Journal of Nonverbal behavior, 24(2), 81-103.

Gleason, J. B. \& Ratner, N. B. (2010). El desarrollo del lenguaje. Madrid: Prentice Hall Pearson Educación.

Hincapié, L., Giraldo, M., Castro, R., Lopera, F., Pineda, D. \& Lopera. E. (2007). Propiedades lingüísticas de los trastornos específicos del desarrollo del lenguaje. Revista Latinoamericana de Psicología, 39(1), 47-61.

Letelier, L. M., Manríquez, J. \& Rada, G. (2005). Revisiones sistemáticas y meta análisis: ¿son la mejor evidencia? Revista Médica de Chile, 133, 246-249.

Lehto, J. E., Juujärvi, P., Kooistra, L. \& Pulkkinen, L. (2003). Dimensions of executive functioning: evidence from children. British Journal of Developmental Psychology, 21(1), 59-80.

Levin, H. S., Song, J., Swing-Cobbs, L. \& Roberson, G. (2001). Porteus maze performance following traumatic brain injury in children. Neuropsychology, 15(4), 557-567. DOI: http://dx.doi. org/10.1037/0894-4105.15.4.557

Lezak, M. D. (1982). The problem of assessing executive functions. International Journal of Psychology, 17(1-4), 281-297. DOI: 10.1080/00207598208247445

López-Ornat, S. (2011). La adquisición del lenguaje, un resumen en 2011. Revista de Investigación en Logopedia, 1(1), 1-11. Recuperado de http:// revistalogopedia.uclm.es

López J. M. \& García, G. C. (2005). Alteraciones lingüísticas en el trastorno especifico del lenguaje. Revista de Neurología, 41(1), 57-63.

Luciana, M. \& Nelson, C. A. (2002). Assessment of neuropsychological function through use of the Cambridge Neuropsychological Testing Automated Battery: performance in 4-to 12-year-old children. Developmental Neuropsychology, 22(3), 595-624. DOI: 10.1207/ S15326942DN2203 3 
Martínez, L., Herrera, C., Valle, J. \& Vásquez, M. (2011). Memoria de trabajo fonológica en preescolares con trastorno especifico del lenguaje expresivo. Revista Psyke, 12(2), 153-162.

Martínez, J. C. \& Romero, R. F. (2007). La emergencia del lenguaje y los sistemas dinámicos. Revista Colombiana de Psicología, 16, 185-202.

Marton, K. (2008). Visuo-spatial processing and executive functions in children with specific language impairment. International Journal of Language \& Communication Disorders, 43(2), 181-200. DOI: 10.1080/16066350701340719

Marton, K., Schwartz, R. G., Farkas, L. \& Katsnelson, V. (2006). Effect of sentence length and complexity on working memory performance in Hungarian children with specific language impairment (SLI): a cross linguistic comparison. International Journal of Language \& Communication Disorders, 41(6), 653-673. DOI: $10.1080 / 13682820500420418$

Marton, K. \& Schwartz, R. (2003). Working memory capacity and language processes in children with specific language impairment. Journal of Speech, Language, and Hearing Research, 46(5), 1138-1153. DOI: 10.1044/10924388(2003/089)

Mauricio, C., Stelzer, F., Mazzoni, C. \& Álvarez, M. A. (2012). Desarrollo de las funciones ejecutivas en niños preescolares. Una revisión de su vínculo con el temperamento y el modo de crianza. Pensando Psicología, 8(15), 128-139.

Mendoza, E. (2012). La investigación actual en el trastorno especifico del lenguaje. Revista de Logopedia, Foniatría y Audiología, 32(2), 75-86. DOI: http://doi.org/10.1016/j.rlfa.2012.03.001

Miyake, A., Friedman, N. P., Emerson, M. J., Witzki, A. H., Howerter, A. \& Wager, T. D. (2000). The unity and diversity of executive functions and their contributions to complex "frontal lobe" tasks: A latent variable analysis. Cognitive psychology, 41(1), 49-100.
Montgomery, J. W. \& Windsor, J. (2007). Examining the language performances of children with and without specific language impairment: contributions of phonological short-term memory and speed of processing. Journal of Speech, Language, and Hearing Research, 50(3), 778797. DOI: 1092-4388/07/5003-0778

Peterson, D. B. \& Gardner, C. M. (2011). Trastornos específicos del lenguaje: una revisión. Revista Chilena de Fonoaudiología, 10, 19-32.

Pineda, D. A. (2000). La función ejecutiva y sus trastornos. Revista de Neurología, 30(8), 764-768.

Pistoia, M., Abad-Mas, L. \& Etchepareborda, M. C. (2004). Abordaje psicopedagógico del trastorno por déficit de atención con hiperactividad con el modelo de entrenamiento de las funciones ejecutivas. Revista de Neurología, 38(1), 149-155.

Quintero, I., Hernández, S., Verche, E., Acosta, V., \& Hernández, A. (2013). Disfunción ejecutiva en el trastorno específico del lenguaje. Revista de Logopedia, Foniatría y Audiología, 33(4), 172-178. DOI: http://doi.org/10.1016/j. rlfa.2013.07.003

Reichenbach, K., Bastian, L., Rohrbach, S., Gross, M. \& Sarrar, L. (2016). Cognitive functions in preschool children with specific language impairment. International Journal of Pediatric Otorhinolaryngology, 86, 22-26. DOI: http://doi. org/10.1016/j.ijporl.2016.04.011

Rodríguez, F. G. (2012). El hermano menor de la palabra. Panorámica de los estudios sobre el gesto. Psiencia. Revista Latinoamericana de Ciencia Psicológica, 4(1), 43-56. DOI: 10.5872/ psiencia/4.1.31

Roebers, C. M. (2006). Developmental progression in children's strategic memory regulation. Swiss Journal of Psychology, 65(3), 193-200. DOI: 10.1024/1421-0185.65.3.193

Rojas-Barahona, C. A. (ed.) (2017). Funciones ejecutivas y educación. Comprendiendo habilidades claves para el aprendizaje. Santiago de Chile: Ediciones UC. 
Santa-Cruz, C. \& Rosas, R. (2017). Mapping of executive functions/Cartografía de las funciones ejecutivas. Estudios de Psicología, 38(2), 284-310.

Von Keyserlingk, L., Castro, P. J. \& Carrasco, J. (2013). Teorías subjetivas de profesionales de escuelas de lenguaje en Chile sobre el trastorno específico del lenguaje. Revista CEFAC, 15(4), 873-883.

Weismer, S. E., Evans, J. \& Hesketh, L. J. (1999). An examination of verbal working memory capacity in children with specific language impairment. Journal of Speech, Language, and Hearing Research, 42(5), 1249-1260.

\section{Recibido: abril 24, 2017}

Aprobado: diciembre 7, 2017 
